\title{
OBSERVATIONS WITH ISOPHOT
}

\author{
D. LEMKE \\ Max-Planck-Institut für Astronomie, Heidelberg, Germany
}

\section{Instrument}

While all channels of the instrument (Lemke et al. 1996) are fully operational in the 21st month of the mission, some have turned out to be particularly productive: (1) far infrared mapping in the $200 \mu \mathrm{m}$ range and (2) spectrophotometry in the middle infrared. The detection sensitivity of these modes is close to the preflight expectation. The photometric calibration of the instrument is a demanding task. Transient and memory effects of the low background detectors, responsivity drifts and spikes caused by the cosmic radiation as well as nonlinearities due to the preamps and the complex optical subsystems require corrections. Fortunately almost all of these effects are reproducable and correction algorithms are under development. While spectrophotometric (Klaas et al. 1997) and staring observations are already calibrated to better than $\pm 20 . .30 \%$ in the pipeline data products, similar values will be achieved for all observing modes in the near future.

\section{Interstellar Dust}

At the longest wavelength the suspected cold galactic cirrus dust has been confirmed as the limiting factor for detection of compact sources for large parts of the sky. The dependence of the fluctuation power on the spatial frequency was extended to values twice as high as the pioneering IRAS investigation at $100 \mu \mathrm{m}$. With the higher spatial resolution the structures break up into ever finer filaments, following the same power law as derived for IRAS's lower spatial frequencies (Herbstmeier et al. 1997). The fine scale structure of the $200 \mu \mathrm{m}$ cirrus seen for the first time behaves similarly. In regions of higher sky brightness $\left(>50 \mathrm{MJy} \mathrm{sr}^{-1}\right)$ the cirrus fluctuations between neighbouring diffraction limited beams reach $\sim 250 \mathrm{mJy}$. In faint regions statistically distributed extragalactic sources cause the noise limit. It is clear from these investigations that deep surveys with large cooled space telescopes and large detector arrays will not suffer from galactic cirrus noise over most of the sky.

The interstellar dust was studied in hitherto inaccessible faint regions: in a nearby cirrus cloud (Lemke et al. 1997), in a quiescent Bok globule (Lehtinen et al. 1997) and as emission from the diffuse matter near the galactic plane (Mattila et al. 1996). All of these regions are far off from hot stars and are heated only by the interstellar radiation field. The high sensitivity of PHT-S and the wide beam capability of the filter photometer made detections possible from the middle to the far infrared. Two interesting results are: (1) the UIR bands are present in a wide variety of objects, their carriers seem to be ubiquitous throughout the Milky Way; (2) the line ratios of $11.3 / 7.7(\mathrm{C}-\mathrm{H} / \mathrm{C}=\mathrm{C})$ vary only slightly, if at all, in contrast to earlier predictions based on the variation of hydrogen coverage of the aromatics. Whether the later findings are explicable by the higher degree of ionization found e.g. in reflection nebulae, or whether they may require a modified model of the suspected carbonaceous carriers, remains unclear at the moment.

It was possible to closely match the energy balance of interstellar dust clouds. A test sample was the Thumbprint Nebula ( $\mathrm{A}_{v} \sim 8 \mathrm{mag}$ ), which shows no signs of star formation and is therefore presumed to be heated only by the interstellar radiation field (Lehtinen et al. 1997). Beyond the reflected light measured in the optical and near infrared, the emission of a $\sim 15 \mathrm{~K}$ dust component at $200 \mu \mathrm{m}$ was mapped for the first time and included in the output. Now the input radiation (UV, optical) matches the sum of the reflected and thermally emitted (FIR) with an accuracy of $\sim 20 \%$.

The sensitive $200 \mu \mathrm{m}$ mapping capability of ISOPHOT has also delivered numerous maps of molecular clouds (fig. 1), where, combined with $100 \mu$ m maps, colour temperature maps have now 
been derived. The coldest knots are found at $\mathrm{T} \sim 12 \ldots 15 \mathrm{~K}$, i.e. the new $200 \mu \mathrm{m}$ observations are imperative because their wavelength is close to the maximum of the emission spectrum. These cold peaks often coincide with supposed preprotostellar condensations (Ward-Thompson et al. 1997, Mattila et al. 1997).

\section{Intergalactic Dust}

The search started in the Coma Galaxy Cluster. Earlier, but controversial, optical observations of galaxies located behind clusters may have indicated an extinction of a few tenths of a magnitude. IRAS measurements of Coma and other clusters gave no detections of dust emission. Two cross shaped scans by ISOPHOT through the centre of Coma at wavelengths 180 and $120 \mu \mathrm{m}$ now indicate an excess of $0.1 \mathrm{MJy} \mathrm{sr}^{-1}$ at the centre (Stickel et al. 1997), see fig 2. At a dust temperature of 20 .. $30 \mathrm{~K}$ this results in a dust mass of $\leq 10^{9} \mathrm{M}_{\odot}$, i.e. a dust/gas ratio of $\sim 1: 10000$. Dust is effectively destroyed on a time scale of $10^{8}$ years in the $8.10^{7} \mathrm{~K}$ plasma environment of cluster gas. But Coma is a young cluster and dust is resupplied by ram stripping from the merging of at least two smaller clusters. Beyond Coma three more clusters are planned to be observed with ISOPHOT to confirm this intracluster dust detection, including an old cluster where no dust detection is expected.

\section{Circumstellar Dust}

The dust disk around Vega discovered by IRAS was mapped by Heinrichsen at al. 1997 using oversampling techniques at 60 and $90 \mu \mathrm{m}$ and photometry up to $200 \mu \mathrm{m}$. They derived disk diameters of 86 and $140 \mathrm{AU}$ for these wavelengths and a mass of the $\mathrm{T} \sim 73 \mathrm{~K}$ material of $\mathrm{M}_{d} \sim 10^{-8} \mathrm{M}_{\odot}$, i.e. a few tenths of the moon's mass. Search for new Vega-like stars (for instance by Ábrahám et al. 1997 in the Ursa Majoris cluster) did not reveal new ones, contrary to predictions that up to $50 \%$ of A-stars might show the phenomenon. The formation of planets out of disks around young stars was studied by Beckwith et al. 1997 in star clusters of different ages. Characteristic and increasing separations between the stellar and the infrared parts of the spectra were discovered in many objects caused by the disks clean up when larger bodies are formed.

\section{Galaxies}

$200 \mu \mathrm{m}$ mapping and photometry have revealed large cold dust masses in both luminous and normal galaxies. Multicolour photometry from $3 \ldots 200 \mu \mathrm{m}$ of interacting galaxies (Klaas et al. 1997) allow dust components in these objects to be separated: a $\sim 140 \mathrm{~K}$ component powered by the starburst and a $\sim 40 \mathrm{~K}$ component heated by the strong interstellar radiation field. A similar bimodal behaviour was discovered for Seyfert galaxies by Rodriguez-Espinosa et al. 1996, except that the hot component is powered by the active nucleus. Genzel et al. 1997 demonstrated that in ultraluminous galaxies the appearance of PAH features $(7.7 \mu \mathrm{m})$ measured with ISOPHOT-S is anticorrelated to the strength of high-ionization lines requiring photon energies of several hundred $\mathrm{eV}$, which at the same time may destroy the PAH line carriers.

High resolution maps at $200 \mu \mathrm{m}$ were obtained from many nearby spiral galaxies (M51, M101, M31, ...). They show surprisingly small temperature variations of the cold dust in spiral arms and the interarm medium (Hippelein et al. 1996, Tufs et al. 1996). A recent map of the Andromeda galaxy at $200 \mu \mathrm{m}$ is of similar spatial resolution to the IRAS $100 \mu \mathrm{m}$ map, but looks different: the nucleus of this spiral almost disappears and the molecular ring and dark clouds dominate. While the nucleus is at $\sim 34 \mathrm{~K}$ the rest of the galaxy is as cold as $\sim 17 \mathrm{~K}$ (Haas et al 1997).

\section{Serendipity Sky Survey}

While slewing from one to the next pointed observation the ISOPHOT C200 camera views the sky. Hereby 3 arcmin wide strip maps are produced along unpredictable curved ("serendipitous") ways, in total $\sim 140000^{\circ}$ long during the mission. They amount to $\sim 500 \mathrm{~h}$ of observing time which would otherwise be lost in slews. About $10 \%$ of the sky will be mapped at this new wavelength range centred at $175 \mu \mathrm{m}$. The huge data base is presently being analysed in two ways: (1) Point like sources are extracted. These are mostly galaxies, a total of $\sim 4000$ can be expected. (2) By 

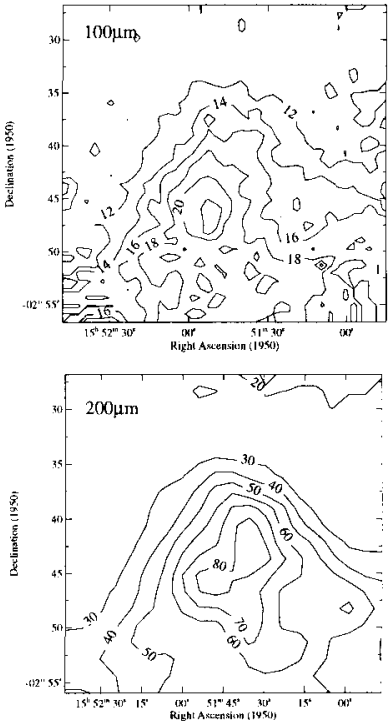

Figure 1. ISOPHOT maps at 100 and $200 \mu \mathrm{m}$ of the dark cloud L183. At the longer wavelength a second peak is seen, its colour temperature is $12 \mathrm{~K}$ and it might indicate a preprotostellar core.
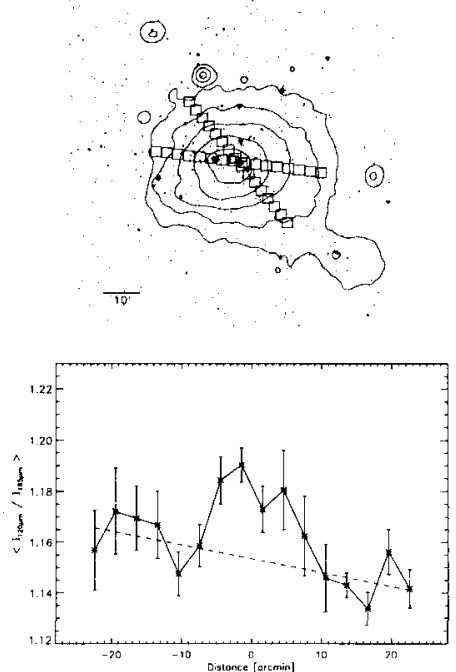

Figure 2. The Coma cluster of galaxies and ROSAT $\mathrm{x}$-ray contours overlaid. The two ISOPHOT scans made at 120 and $185 \mu \mathrm{m}$ are indicated. Their ratios across the cluster indicate an excess, interpreted as dust emission from the central region.

correlating the ISOPHOT strip maps to the IRAS $100 \mu \mathrm{m}$ "road" maps, colour temperatures can be immediately derived for the cirrus and molecular cloud emission on a full sky scale.

The detailed analysis of a selected $10^{\circ} \times 10^{\circ}$ field near the ecliptic north pole ("minisurvey") has given several indications of the wealth of results to be expected from the total survey (Bogun et al. 1998): (i) more than 20 point-like sources were found, resulting in about 1 detection per $40^{\circ}$ slew length, (ii) $\sim 70 \%$ of these sources are spiral galaxies including mergers, (iii) almost all brighter ( $>2$ Jy) sources have IRAS $100 \mu \mathrm{m}$ counterparts, (iv) the detection limit can be expected at 1..1.5 Jy depending on the hit geometry. The full slew evaluation in the Ophiuchus molecular cloud revealed dust temperatures of between 12 and $26 \mathrm{~K}$, where the coldest spots can be correlated to radio and molecular line maps with an accuracy of $\sim 1$ arcmin.

I acknowledge the support throughout the project of DARA, Bonn, the Max-Planck Society, my colleages in the international ISOPHOT consortium, the ISOPHOT Data Centre at MPIA, Heidelberg, the PHT Instrument Dedicated Team and ESA's Science Operations Centre, VILSPA.

\section{References}

Ábrahám, P., Leinert, C., Lemke, D. et al, in Conf. Proc.: "ISO's View on Stellar Evolution", Noordwijkerhout, 1997 Beckwith, S.W.B. et al., 1998, in preparation

Bogun, S., Stickel, M., Lemke, D. et al., 1998, A\&A, to be submitted

Genzel, R., Lutz, D., Sturm, E., et al., 1998, ApJ, submitted

Haas, M., Lemke, D., Stickel, M. et al., 1997, in preparation

Heinrichsen, I., Walker, H.J., Klaas, U. and Beckwith, S.W.B., 1997, Mon. Not. R. Astron. Soc., in press Hippelein, H., Lemke, D., Tuffs, R. et al., 1996, A\&A315, L79

Klaas, U., Acosta-Pulido, J.A., Ábrahám, P. et al., in Conf. Proc. "ISO spectroscopy", VILSPA, 1997, in press

Klaas, U., Haas, M., Heinrichsen, I. and Schulz, B., 1997, A\&A325, L21

Lehtinen, K., Lemke, D., Mattila, K. and Haikala, L., 1997, A\&A, in press

Lemke, D., Klaas, U., Abolins, J. et al., 1996, A\&A315, L64

Lemke, D., Mattila, K., Lehtinen, K. et al., 1997, A\&A, in press

Mattila, K, Lemke, D., Haikala, L et a], 1996, A\&A315, L353

Mattila, K., Lemke, D. and Haikala, L., in Conf.Proc.: "Star Formation with ISO", Lisbon, 1997

Rodriguez-Espinosa, J.M., Perez Garcia, A.M., Lemke, D. et al., 1996, A\&A315, L129

Stickel, M. Lemke, D., Mattila, K. Haikala, L.K. and Haas, M., 1997, A\&A, in press

Tuffs, R.J., Lemke, D., Xu, C. et al., 1996, A\&A315, L149

Ward-Thompson et al., in Conf.Proc.: "Star Formation with ISO", Lisbon, 1997 